\title{
Social networks, sedentary behavior and physical activity
}

\begin{abstract}
Using self-determination theory (SDT) as a theoretical framework, the primary aim of this mini-review was to identify the relationships among perceptions of supportive social networks (physical activity instructors' support, family support, and peer support), psychological need satisfaction (autonomy, competence, and relatedness), physical activity motivation, sedentary behavior and physical activity in aging population. Perceptions of supportive social networks should facilitate older adults' motivation and ultimately promote their leisure-time physical activity and reduce their sedentary behavior.
\end{abstract}

Keywords: self-determination theory, supportive environment, motivation, sedentary behavior, physical activity developing supportive social networks to promote older adults' physical activity and reduce sedentary behaviors: a self-determination theory perspective
Volume 3 Issue 5 - 2018

Tao Zhang, Xiangli Gu,Tsz Lun (Alan) Chu

Department of Kinesiology, University of North Texas, USA

Correspondence: Tao Zhang, Department of Kinesiology, Health Promotion and Recreation, University of North Texas, Denton, Texas, 76203-5017, USA, Tel 940-565-3415, EmailTao.Zhang@unt.edu

Received: December 26, 2017 | Published: October 01, 2018

\section{Introduction}

There is an increasing proportion of older adults across the world. It was reported that these older adults aged 65 years and above will constitute $16 \%$ of the global population by 2050 (World Health Organization [WHO], 2016). ${ }^{1}$ Physical activity is critical to retaining health, but a majority of older adults are insufficiently physically active and have high levels of sedentary (sitting) time., ${ }^{2,3}$ It is well documented that sedentary behaviors among aging populations are associated with health problems such as diabetes, cardiovascular disease and death. ${ }^{2}$ The U.S. Department of Health and Human Services $^{4}$ recommends that older adults should engage in at least 150 minutes of moderate intensity physical activity per week in bouts of at least 10 minutes each, or 75 minutes of vigorous intensity activity, or an equivalent combination of these two, per week, in addition to balance (three times per week) and muscle strengthening (twice per week) activities.

Based on the notion that supportive social networks can effectively promote older adults' regular leisure-time physical activity, and that engagement in regular physical activity during aging can positively influence their health and well-being, 4,5 it is important to foster older adults' motivation toward physical activity in order to reduce sedentary behavior. Unfortunately, little is known about the health consequences of changes in social networks and its influences on sedentary behaviors and their motivation toward physical activity. Identifying theory-based motivational factors that positively influence physical activity behavior has the potential to provide a clearer understanding of how older adults make decisions to be physically active. ${ }^{6,7}$ This effort also can facilitate the development of effective intervention strategies aimed at the prevention of physical inactivity and sedentary behavior in aging populations.

Self-determination theory ${ }^{8-10}$ is a promising theoretical framework used to explain individuals' motivation and behaviors, and thus may enhance our understanding of older adults' motivation to engage in physical activity. According to SDT, an individual's motivation is not a direct function of social environment (e.g. social support from physical activity instructors, family members, and peers). Rather, SDT assumes that the impact of social environment is mediated by basic psychological needs (i.e., needs for autonomy, competence, and relatedness; Deci et al. ${ }^{8}$ ). Specifically, the need for autonomy is satisfied if individual feels self-determined in his/her actions rather than feeling controlled or obliged to act. The need for competence is satisfied through the pursuit of autonomously motivated behaviors that lead to perceptions of success and control of outcomes. The need for relatedness is satisfied through innate desires to be socially supported by others and be supportive of others when engaging in behaviors. ${ }^{10,11}$ A social network that meets and satisfied all three basic psychological needs should promote an individual's optimal motivation and healthy behaviors such as physical activity. ${ }^{11,12}$

SDT proposes that satisfaction of the needs for autonomy, competence, and relatedness (i.e., psychological need satisfaction) facilitates self-determined motivation and is central to motivational outcomes. ${ }^{8}$ Whereas self-determined motivation represents an adaptive form of motivation that individuals internalize and integrate external values and experiences Deci \& Ryan $^{8,9}$ argue that people do not always do so. In such circumstances, people are less self-determined, but rather more controlled or even amotivated. Deci \& Ryan ${ }^{8,9}$ conceptualize a self-determination continuum to distinguish six types of motivations with different degrees of self-determination, ranging from intrinsic motivation (most self-determined) to amotivation (least self-determined). Extrinsic motivation with decreasing levels of selfdetermination integrated regulation, identified regulation, introjected regulation and external regulation fall between these two extremes on the continuum.

Based on SDT, 9,10 the most desirable and long lasting level of motivation is intrinsic motivation. Intrinsic motivation refers to highly autonomous behaviors whereby individual engages in physical activity for the inherent feeling of pleasure, accomplishment, and the experience of sensations. When individuals are intrinsically motivated, they choose to engage in an activity for its own sake rather than for an external reason. That is, intrinsic motivation is considered to be the most desirable regarding adherence because physical 
activity is based on appreciation of the activity itself rather than appreciation for some benefits provided by the activity. In contrast, extrinsic motivation refers to activities that are carried out as a means to an end that is valued (i.e., praise, extrinsic reward) and not for the sake of the activity itself. According to SDT, extrinsic motivation is multidimensional in nature and comprises four dimensions: integrated regulation, identified regulation, introjected regulation and external regulation. Integrated regulation, the most self-determined form of extrinsic motivation, refers to behaviors that are performed out of choice to harmonize and bring coherence to different parts of the self. Identified regulation refers to behaviors that are highly valued and performed out of choice. This type of extrinsic motivation reflects an internalized and self-initiated behavior, even though the behavior itself is not always fun. Introjected regulation refers to engaging in behaviors in order to achieve social recognition, or avoid internal pressures and feelings of guilt, or to please significant others. The last type of extrinsic motivation, external regulation, represents the lowest degree of self-determination among the four forms of extrinsic motivation and refers to situations in which individuals complete an activity in order to attain tangible rewards or to avoid punishment. Beyond intrinsic motivation and extrinsic motivation, Deci \& Ryan ${ }^{8}$ argue that individuals can also be amotivated toward an activity. Amotivation occurs when individuals experience a lack of contingency between their behavior and outcomes, and it represents a complete absence of self-determination and volition with respect to a targeted behavior. This is evident when individuals do not value an activity at all, or when they experience feelings of incompetence and uncontrollability. According to previous findings, unfortunately, many older adults lack self-determined motivation to be physically active and adopt a sedentary lifestyle. ${ }^{2,7}$ Different types of motivation most likely play a role in the mechanism that leads to regular physical activity in this population.

Although sufficient evidence has shown that SDT provides a useful conceptual basis for understanding why people intend to continue with motivational behaviors in the physical activity and exercise domain, ${ }^{9}$ little empirical research has investigated the influences of multifaceted social networks, such as social support from physical activity instructors, family members, and peers, on older adults' motivation and subsequent physical activity and sedentary behavior. ${ }^{6,7}$ Thus, more investigations are needed to explore the effects of multifaceted social networks on older adults' sedentary behavior and physical activity, with psychological need satisfaction and motivation being tested as potential mediators. Thus, by adopting a hierarchical mode of intrinsic and extrinsic motivation based on SDT, ${ }^{12}$ it may be possible to elucidate the motivational process through which multifaceted social networks influence need satisfaction, motivation and engagement in health-related behavior, such as sedentary behavior and physical activity. Identifying the mediating effects of need satisfaction and motivation on leisure-time sedentary behavior and physical activity using structural equation modeling may facilitate our understanding of supportive social networks on positive physical activity behavior among older adults.

\section{Conclusion}

promoting older adults' leisure-time physical activity and reducing their sedentary behavior are research priorities in the field. Researchers and practitioners need to consider the multifaceted social networks (physical activity instructors' support, family support, and peer support) as they design interventions to promote physical activity and reduce sedentary behavior among older adults. To identify the effects of multifaceted social networks on older adults' sedentary behavior and physical activity, it is also imperative to examine the mediating effects of need satisfaction and motivation using self-determination theory as a theoretical framework. Such effort can facilitate the development of effective intervention strategies aimed at the prevention of sedentary behavior in aging populations.

\section{Acknowledgements}

None.

\section{Conflict of interest}

Author declares that there is no conflict of interest.

\section{References}

1. http://www.who.int/ageing/publications/global_health.pdf

2. Copeland JL, Ashe MC, Biddle SJ, et al. Sedentary time in older adults: A critical review of measurement, associations with health, and interventions. Br J Sports Med. 2017;51(21):1539-1547.

3. Schlaff, RA, Baruth M, Boggs A, et al. Patterns of sedentary behavior in older adults. Am J Health Behav. 2017;41(4):411-418.

4. https://health.gov/paguidelines/guidelines/

5. https://www.healthypeople.gov/

6. Cornwell B, Laumann EO. The health benefits of network growth: New evidence from a national survey of older adults. Soc Sci Med. 2015;125:94-106

7. Zhang T, Solmon A, Kosma M, et al. Need support, need satisfaction, intrinsic motivation, and physical activity participation among middle school students. Journal of Teaching in Physical Education. 2011;30:51-68.

8. Deci EL, Ryan RM. Intrinsic motivation and self-determination in human behaviour. New York; 1985.

9. Ryan RM, Deci EL. Self-determination theory: Basic psychological needs in motivation, development, and wellness. Guilford, New York; 2017

10. Deci EL, Ryan RM. The "what" and "why" of goal pursuits: Human needs and the self-determination of behavior. Psychological Inquiry. 2000;11(4):227-268.

11. Ryan RM, Deci EL. Overview of self-determination theory: An organismic dialectical perspective. In: EL Deci \& RM Ryan editors, Handbook on self-determination research. Rochester, NY: University of Rochester Press. 2002:3-33.

12. Vallerand RJ. Deci and Ryan's self-determination theory: A view from the hierarchical model of intrinsic and extrinsic motivation. Psychological Inquiry. 2000;11(4):312-318. 